\title{
8.6. РАННЕЕ ПРЕДПРИНИМАТЕЛЬСТВО В ТРЕТЬЕМ ВОЗРАСТЕ
}

\author{
Пиньковецкая Ю.С., к.э.н., доцент кафредры экономического анализа и государственного управления
}

Ульяновский государственный университет, 2. Ульяновск

В связи с изменением пенсионного возраста в Российской Федерации значимость предпринимательства в третьем возрасте будет повышаться. Целью статьи является анализ особенностей, закономерностей и тенденций, которые характеризуют раннее предпринимательство населения в третьем возрасте. Создание своих бизнесов может использоваться гражданами для продления трудовой жизни, улучшения финансового положения по сравнению с наемным трудом, отсрочки выхода на пенсию. В статье представлен анализ литературы по проблеме ранней предпринимательской активности в экономически развитых странах.

\section{Литература}

1. Численность и состав населения [Электронный ресурс] // Федер. служба гос. статистики. - 2019. - URL : http://www.gks.ru/wps/wcm/connect/rosstat_main/rosstat/ru/statistics/population/demography/.

2. Ahmad N.H. et al. Silver entrepreneurship agenda in Malaysia: a proposed model for productive aging [Text] / N.H. Ahmad, A.M. Nasurdin, H.A. Halim, P. Seet // The Winners. - 2012. - No. 13. - Pp. 1-8.

3. Botham R. Third age entrepreneurs: innovative business start ups in mid-life and beyond- understanding the drivers and removing the barriers [Text] / R. Botham, A. Graves // Interim report to NESTA. - 2009. - Feb.

4. Cressy R. New firms and their banks [Text] / R. Cressy, D. Storey. - Warwick university business schooland NatWest. - 1995.

5. Fairlie R.W. Kaufman index of entrepreneurial activity, 1996-2013 [Electronic resource] / R.W. Fairlie. - 2014. URL : https://escholarship.org/content/qt8nx5s6b1/qt8nx5s6b1.pdf.

6. Fuertes V. et al. Extending working lives: age management in SMEs [Text] / V. Fuertes, V. Egdell, R. McQuaid // Employee relations. -2013 . - No. 35. - Pp. 272-293.

7. Gielnik M.M. Age in the entrepreneurial process: the role of future time perspective and prior entrepreneurial experience [Text] / M.M. Gielnik, H. Zacher, M. Wang // J. applied psycholgies. - 2018. - No. 103. - Pp. 1067-1085.

8. Gimmon E. et al. Entrepreneurship in the third age: retirees' motivation and intentions [Text] / E. Gimmon, R. Yitshaki, S. Hantman // International j. entrepreneurship and small business. - 2018. - Vol. 34 ; no. 3. - Pp. 267-288.

9. Gordon J.K. Older is wiser? It depends who you ask... and how you ask [Text] / J.K. Gordon, L.M. Jordan // Aging neuropsychology and cognition. - 2016. - №24. - Pp. 1-21.

10. Havighurst R.J. Successful aging [Text] / R.J. Havighurst // Williams R.H. et al. Processes of aging. - Atherton press, New York, 1963. - Pp. 299-320.

11. Kautonen T. Understanding the older entrepreneur: comparing third age and prime age entrepreneurs in Finland [Text] / T. Kautonen // International j. of business science and applied management. -2008 . - No. 3. - Pp. 3-13.

12. Kautonen T. et al. Enterprise support for older entrepreneurs: the case of PRIME in the UK [Text] / T. Kautonen, S. Down, L. South // International j. of entrepreneurial behavior \& research. - 2008. - Vol. 14 ; no. 2. - P. 85-101.

13. Kautonen T. et al. Entrepreneurial intentions in the third age: the impact of perceived age norms [Electronic resource] / T. Kautonen, E.T. Tornikoski, E. Kibler // Small business economics. - 2009. - Vol. 37 ; no. 2. - Pp. 219-234. - URL : http://link.springer.com/10.1007/s11187-009-9238-y.

14. Kautonen T. et al. Predicting entrepreneurial behaviour: a test of the theory of planned behavior [Text] / T. Kautonen, M. van Gelderen, E.T. Tornikoski // Applied economics. - 2011. - Vol. 45 ; no. 6. - Pp. 697-707.

15. Kautonen T. Do age-related social expectations influence entrepreneurial activity in later life? [Text] / T. Kautonen // The international j. of entrepreneurship and innovation. - 2012. - Vol. 13 ; no. 3. - Pp. 179-187.

16. Kautonen T. et al. Ageing and entrepreneurial preferences [Text] / T. Kautonen, S. Down, M. Minniti // Small business economics. - 2013. - Vol. 42 ; no. 3. - Pp. 579-594.

17. Kibler E. et al. Under pressure: differentiation in older entrepreneur motivations, emotions and mentoring [Text] / E. Kibler, T. Wainwright, R. Blackburn, T. Kautonen // 35th Institute for small business and entrepreneurship (ISBE) annual conference. - Dublin, IE., 2012.

18. Kibler E. et al. Can social exclusion against "older entrepreneurs" be managed? [Text] / E. Kibler, T. Wainwright, T. Kautonen, R. Blackburn // J. of small business management. - 2015. - No. 53. - Pp. 193-208.

19. Kirkwood J. Motivational factors in a push-pull theory of entrepreneurship [Text] / J. Kirkwood // Gender in management. 2009. - Vol. 24 ; no. 5. - Pp. 346-364.

20. Levesque M. The effect of aging on entrepreneurial behavior [Text] / M. Levesque, M. Minniti // J. of business venturing. 2006. - Vol. 21 ; no. 2. - Pp. 177-194.

21. Loretto W. Work and retirement in an ageing world: the case of older workers in the UK [Text] / W. Loretto // J. of the Academy of social sciences. -2010 . - Vol. 5 ; no. 3. - Pp. 279-294.

22. Loretto W. et al. Older workers and options for flexible work [Text] / W. Loretto, S. Vickerstaff, P. White. - Manchester : Equal opportunities commission, 2005.

23. Loretto W. Employers attitudes, practices and policies towards older workers [Text] / W. Loretto, P. White // Human resource management j. - 2006. - Vol. 16 ; no. 3. - Pp. 313-330.

24. Radford K. et al. What makes me stay? An investigation into factors influencing older workers' intentions to stay [Text] / K. Radford, K. Shacklock, E. Meissner // Labour \& industry: a journal of the social and economic relations of work. - 2015. No. 25. - Pp. 306-320.

25. Singh G. Early retirees as the next generation of entrepreneurs [Text] / G. Singh, A. DeNoble // Entrepreneurship theory and practice. - 2003. - Vol. 27 ; no. 3. - Pp. 207-226.

26. Stamov-Roßnagel C. Ageing and work motivation: a task-level perspective [Text] / C. Stamov-Roßnagel, T. Biemann // J. of 27. managerial psychology. - 2012. - Vol. 27 ; no. 5. - Pp. 459-478. 
28. Wainwright T. et al. Who are you calling old? [Electronic resource] / T. Wainwright, E. Kibler, R. Blackburn, T. Kautonen // Revisiting notions of age and ability amongs older entrepreneurs. - 2011 . URL https://eprints.kingston.ac.uk/21480/1/Wainwright-T-21480.pdf.

29. Zhang T. Elderly entrepreneurship in an aging US economy: it's never too late [Text] / T. Zhang // World scientific. -2008.

30. Weber P. Understanding the grey entrepreneur [Text] / P. Weber, M. Schaper // J. of enterprising culture. - 2004. - Vol. 12 ; no. 2. - Pp. 147-164.

31. Webster B.J. et al. Smart training for the older entrepreneur [Text] / B.J. Webster, E.A. Walker, J. Turner // Paper presented at the ICSB 2005 World Conference. Melbourne, June 2005.

32. Walker E.A. Gender, age and self-employment: some things change, some stay the same [Text] / E.A. Walker, B.J. Webster // Women in management review. - 2007. - Vol. 22 ; no. 2. - Pp. 122-135.

\section{Ключевые слова}

Ранее предпринимательство; третий возраст; экономически развитые страны; мотивация предпринимательства; индивидуальное предпринимательство; психологические барьеры; предпринимательская активность; человеческий капитал; пенсионный возраст; изменение структуры населения.

\section{Пиньковецкая Юлия Семеновна}

\section{РЕЦЕНЗИЯ}

В связи с увеличением пенсионного возраста и Российской Федерации и старением населения страны актуальной становится проблема обеспечения занятости жителей предпенсионного и пенсионного возраста. Одним из наиболее важных направлений обеспечения занятости людей в возрасте от 50 лет и старше является предоставление возможностей проявления ими предпринимательской активности, те. создания малых предприятий и развития индивидуального предпринимательства. Реализацией этой стратегии является развитие женского предпринимательства, которому до настоящего времени не уделялось достаточного внимания в государственной политике поддержки предпринимательского сектора РФ. Учитывая это, значительный интерес представляет анализ закономерностей и тенденций ранней предпринимательской активности населения старшего возраста.

В статье рассматриваются результаты научных исследований, выполненных учеными, по информации, характеризующей накопленный в экономически развитых странах опыт по указанной проблеме. Особое внимание уделяются положительным и негативным аспектам раннего предпринимательства в пожилом возрасте, мотивации таких предпринимателей.

Приведенные в статье положения хорошо аргументированы. Автором подробно проанализирован ряд наиболее важных для понимания рассматриваемой проблемы исследований. Статья написана доступно и логично, термины используются корректно. Приведенная в статье информация представляет значительный интерес для начинающих предпринимателей в нашей стране, федеральных, региональных и муниципальных органов управления, связанных с поддержкой предпринимательства.

Заключение: содержание рецензируемой статьи соответствует требованиям, предъявляемым к научным статьям, и она может быть рекомендована к изданию в журнале «Аудит и финансовый анализ».

Лапин А.Е., д.э.н., профессор, заведующий кафедрой экономического анализа и государственного управления, Ульяновский государственный университет, г. Ульяновск.

DOI 10.38097/AFA.2020.12.69.030 